\title{
EULER-STIELTJES CONSTANTS FOR THE RANKIN-SELBERG $L$-FUNCTION AND WEIGHTED SELBERG ORTHOGONALITY
}

\author{
Almasa Odžak and Lejla Smajlović
}

University of Sarajevo, Bosnia and Herzegovina

\begin{abstract}
Let $E$ be Galois extension of $\mathbb{Q}$ of finite degree and let $\pi$ and $\pi^{\prime}$ be two irreducible automorphic unitary cuspidal representations of $G L_{m}\left(E_{\mathbb{A}}\right)$ and $G L_{m^{\prime}}\left(E_{\mathbb{A}}\right)$, respectively. We prove an asymptotic formula for computation of coefficients $\gamma_{\pi, \pi^{\prime}}(k)$ in the Laurent (Taylor) series expansion around $s=1$ of the logarithmic derivative of the Rankin-Selberg $L$-function $L\left(s, \pi \times \tilde{\pi}^{\prime}\right)$ under assumption that at least one of representations $\pi, \pi^{\prime}$ is self-contragredient and show that coefficients $\gamma_{\pi, \pi^{\prime}}(k)$ are related to weighted Selberg orthogonality. We also replace the assumption that at least one of representations $\pi$ and $\pi^{\prime}$ is self-contragredient by a weaker one.
\end{abstract}

\section{INTRODUCTION}

The classical Euler constant

$$
\gamma=\lim _{x \rightarrow \infty}\left(\sum_{n<x} \frac{1}{n}-\log x\right)=0.57721 \ldots
$$

discovered and computed correctly up to five decimal places by L. Euler ([7]) in 1731 is the constant term in the Laurent series expansion of the Riemann zeta function at $s=1$

$$
\zeta(s)=\frac{1}{s-1}+\gamma+\sum_{k=1}^{\infty} \gamma_{k}(s-1)^{k}=\frac{1}{s-1}+\sum_{k=0}^{\infty} \gamma_{k}(s-1)^{k}
$$

2010 Mathematics Subject Classification. 11M26, 11S40.

Key words and phrases. Euler-Stieltjes constants, Rankin-Selberg $L$-function, weighted Selberg orthogonality. 
In 1885, T. J. Stieltjes ([14]) proved that

$$
\gamma_{k}=\frac{(-1)^{k}}{k !} \lim _{x \rightarrow \infty}\left(\sum_{n<x} \frac{\log ^{k} n}{n}-\frac{\log ^{k+1} x}{k+1}\right) .
$$

Therefore, the constants $\gamma_{k}(k \geq 0)$ with $\gamma_{0}=\gamma$ are named the Stieltjes constants, the generalized Euler constants or the Euler-Stieltjes constants.

The Euler-Stieltjes constants $\gamma_{k}$ can be expressed in terms of coefficients $\eta_{k}$ of the Laurent series expansion of the logarithmic derivative of the Riemann zeta function at $s=1$

$$
\frac{\zeta^{\prime}}{\zeta}(s)=-\frac{1}{s-1}+\sum_{k=0}^{\infty} \eta_{k}(s-1)^{k},|s-1|<3 .
$$

Constants $\eta_{k}$ can be evaluated as

$$
\eta_{k}=\frac{(-1)^{k-1}}{k !} \lim _{x \rightarrow \infty}\left(\sum_{n<x} \frac{\Lambda(n) \log ^{k} n}{n}-\frac{\log ^{k+1} x}{k+1}\right),
$$

where $\Lambda(n)$ is the von Mangoldt function, see e.g. [37]. Often, constants $\gamma_{k}$ are called the Euler-Stieltjes constants of the first kind, while constants $\eta_{k}$ are called the Euler-Stieltjes constants of the second kind.

Throughout this paper, we will slightly abuse the notation and denote the Euler-Stieltjes constants of the second kind (i.e. constants appearing in the Laurent (Taylor) series representation of the logarithmic derivative of the corresponding $L$-series) by letter $\gamma$ instead of $\eta$.

The Euler-Stieltjes constants of the first and the second kind are important in both theoretical and computational analytic number theory, since they appear in various estimations and as a result of asymptotic analysis. For example, the Euler-Stieltjes constants of the second kind can be used to determine a zero-free region of the zeta function near the real axis in the critical strip. Moreover, they are related to the Li (positivity) criterion for the Riemann hypothesis $([20])$, since they appear in arithmetic formula for the non-archimedean part of the Li coefficient, see [23] for numerical evaluation and estimation. The Euler-Stieltjes constants of the first and the second kind and their relation to the Li criterion for the Riemann hypothesis were further investigated by M. Coffey in [4,5] and by C. Knessl and M. Coffey in [19].

The generalized Euler-Stieltjes constants of the second kind, i.e. the coefficients in the Laurent (Taylor) series expansion of logarithmic derivative of zeta or $L$-function at $s=1$ were subject of study in many different settings, see e.g. [3] for the Hurwitz zeta function, [13] for the Dedekind zeta function and the Selberg zeta function attached to a co-compact Fuchisian group, [1] for the general setting of a non-co-compact Fuchsian group with unitary representation, [12] for a class of functions that have an Euler product representation converging in some half-plane and a simple pole on the real axes. 
For functions $F$ that belong to a large subclass $\mathcal{S}^{b}$ of the Selberg class it is proved that the coefficients $\gamma_{F}(k)$ in the Laurent (Taylor) series expansion of $\frac{F^{\prime}}{F}(s)$ at $s=1$ can be expressed as

$$
\gamma_{F}(k)=\frac{(-1)^{k-1}}{k !} \lim _{x \rightarrow \infty}\left(\sum_{n<x} \frac{\Lambda_{F}(n) \log ^{k} n}{n}-\frac{m_{F} \log ^{k+1} x}{k+1}\right),
$$

where $\Lambda_{F}(n)$ is generalized von Mangoldt function defined in terms of the coefficients in the Euler product of $F$ and $m_{F}$ is the order of (eventual) pole of $F \in \mathcal{S}^{b}$ at $s=1$, see [36, Corollary 6.3.].

Let $E$ be a Galois extension of $\mathbb{Q}$ of a finite degree and let $\pi$ and $\pi^{\prime}$ be irreducible unitary cuspidal representations of $G L_{m}\left(E_{\mathbb{A}}\right)$ and $G L_{m^{\prime}}\left(E_{\mathbb{A}}\right)$, respectively. The generalized Euler-Stieltjes constants of the second kind $\gamma_{\pi, \pi^{\prime}}(k)$ attached to the Rankin-Selberg $L$-function $L\left(s, \pi \times \tilde{\pi}^{\prime}\right)$ are defined as coefficients in the Laurent series representation of the logarithmic derivative of $L\left(s, \pi \times \tilde{\pi}^{\prime}\right)$ at $s=1$ :

$$
\frac{L^{\prime}}{L}\left(s, \pi \times \widetilde{\pi}^{\prime}\right)=\sum_{k=-1}^{\infty} \gamma_{\pi, \pi^{\prime}}(k)(s-1)^{k} .
$$

In this paper we derive an asymptotic formula for evaluation of constants $\gamma_{\pi, \pi^{\prime}}(k)$, under assumption that at least one of representations $\pi$ or $\pi^{\prime}$ is self-contragredient.

The Rankin-Selberg $L$-function $L\left(s, \pi \times \tilde{\pi}^{\prime}\right)$ does not always belong to the Selberg class (nor to the class of functions considered in [12]) since, in the case when $m=m^{\prime}$ and $\pi^{\prime} \cong \pi \otimes|\operatorname{det}|^{i t_{0}}$, for some $t_{0} \in \mathbb{R} \backslash\{0\}$ it possesses pole at $s=1+i t_{0} \neq 1$. In this setting, it is possible to normalize representations $\pi$ and $\pi^{\prime}$ so that the "normalized" Rankin-Selberg $L$-function possesses a pole at $s=1$, hence computation of coefficients in the Laurent series expansion of its logarithmic derivative at $s=1$ reduces to a slight modification of the case treated in [36].

However, in the case when $m=m^{\prime}$ and $\pi^{\prime} \cong \pi \otimes|\operatorname{det}|^{i t_{0}}$, for some $t_{0} \in \mathbb{R} \backslash\{0\}$, it is actually of interest to compute coefficients $\gamma_{\pi, \pi^{\prime}}(k)$ (of the Taylor series expansion (1.1) of $L\left(s, \pi \times \tilde{\pi}^{\prime}\right)$ at $\left.s=1\right)$, since they appear in various arithmetic formulas. For example, coefficients $\gamma_{\pi, \pi^{\prime}}(k)$ appear in the arithmetic formula for the Li coefficients attached to $L\left(s, \pi \times \widetilde{\pi}^{\prime}\right)$ that is used to formulate the Li criterion for the generalized Riemann hypothesis for the Rankin-Selberg convolution in [27]. Expressing coefficients $\gamma_{\pi, \pi^{\prime}}(k)$ in terms of the Satake and Langlands parameters related to $\pi$ and $\pi^{\prime}$ enables one to relate the generalized Riemann hypothesis to non-negativity of sequence of numbers expressed only in terms of arithmetic parameters related to corresponding representations, see Corollary 3.5. below.

Furthermore, the coefficients $\gamma_{\pi, \pi^{\prime}}(k), k \geq 0$ are closely related to the weighted Selberg orthogonality, since $\gamma_{\pi, \pi^{\prime}}(k), k \geq 0$ are constant terms in 
the asymptotic expansion of the sum

$$
\sum_{n \leq x} \frac{\Lambda_{\pi, \pi^{\prime}}(n) \log ^{k} n}{n}
$$

as $x \rightarrow \infty$, where $\Lambda_{\pi, \pi^{\prime}}(n)$ are coefficients in the Dirichlet series representation of logarithmic derivative of $L\left(s, \pi \times \tilde{\pi}^{\prime}\right)$, see formula (2.1) below.

As a corollary, assuming generalized form of the Hypothesis $\mathrm{H}$ of Rudnick and Sarnak ([28]) posed in [10] in the case when $m$ or $m^{\prime}$ is greater than four, we deduce the Selberg orthogonality conjecture for irreducible unitary cuspidal representations $\pi$ and $\pi^{\prime}$ of $G L_{m}\left(E_{\mathbb{A}}\right)$ and $G L_{m^{\prime}}\left(E_{\mathbb{A}}\right)$.

In the last section, we show that the main result of the paper holds true if we replace the assumption that at least one of representations $\pi$ or $\pi^{\prime}$ is self-contragredient by a weaker one.

\section{Preliminaries}

2.1. Rankin-Selberg L-functions. Let $E$ be a Galois extension of $\mathbb{Q}$ of degree $l$, and let $E_{\mathbb{A}}$ denote the ring of adeles over $E$. For a finite place $v$, let $f_{p}$ denote the modular degree of $E_{v}$ over $\mathbb{Q}_{p}$ for $v \mid p$. The automorphic $L$-function attached to the irreducible cuspidal representation $\pi$ of $G L_{m}\left(E_{\mathbb{A}}\right)$ with unitary central character is defined for Res $>1$ as the absolutely convergent product of its local factors $L(s, \pi)=\prod_{v<\infty} L_{v}\left(s, \pi_{v}\right)$. In the case when $\pi_{v}$ is unramified

$$
L_{v}\left(s, \pi_{v}\right)=\prod_{j=1}^{m}\left(1-\alpha_{\pi}(v, j) p^{-f_{p} s}\right)^{-1},
$$

where $\alpha_{\pi}(v, j)$ denote Satake parameters. The local factors at ramified places $v$ can be written in the same form, with the convention that some of $\alpha_{\pi}(v, j)$ may be zero.

For $\operatorname{Re} s>1$, the logarithmic derivative of $L(s, \pi)$ can be written as a Dirichlet series

$$
\frac{L^{\prime}}{L}(s, \pi)=-\sum_{n=1}^{\infty} \frac{\Lambda(n) \alpha_{\pi}(n)}{n^{s}},
$$

where $\Lambda(n)$ is the von Mangoldt function and

$$
\alpha_{\pi}\left(p^{k f_{p}}\right)=\sum_{v \mid p} f_{p} \sum_{j=1}^{m} \alpha_{\pi}(v, j)^{k} .
$$

The local factors at infinite places are

$$
L_{v}\left(s, \pi_{v}\right)=\prod_{j=1}^{m} \Gamma_{v}\left(s+\mu_{\pi}(v, j)\right),
$$


where $\left\{\mu_{\pi}(v, j)\right\}_{j=1}^{m}$ are the Langlands parameters associated to $\pi_{v}$ and $\Gamma_{v}(s)=\pi^{-s / 2} \Gamma(s / 2)$, if $v$ is real and $\Gamma_{v}(s)=2(2 \pi)^{-s} \Gamma(s)$, if $v$ is complex.

Analogously, the (finite part) Rankin-Selberg $L$-function attached to the product $\pi \times \widetilde{\pi}^{\prime}$ of irreducible cuspidal representations of $G L_{m}\left(E_{\mathbb{A}}\right)$ and $G L_{m^{\prime}}\left(E_{\mathbb{A}}\right)$ with unitary central character, respectively, is given, for Res $>1$, by the absolutely convergent Euler product of local factors $L\left(s, \pi \times \widetilde{\pi}^{\prime}\right)=$ $\prod_{v<\infty} L_{v}\left(s, \pi_{v} \times \widetilde{\pi}_{v}^{\prime}\right)$, see e.g. [15], where

$L_{p}\left(s, \pi \times \tilde{\pi}^{\prime}\right)=\prod_{v \mid p} L_{v}\left(s, \pi_{v} \times \widetilde{\pi}_{v}^{\prime}\right)=\prod_{v \mid p} \prod_{j=1}^{m} \prod_{k=1}^{m^{\prime}}\left(1-\alpha_{\pi}(v, j) \overline{\alpha_{\pi^{\prime}}(v, k)} p^{-f_{p} s}\right)^{-1}$

and $\widetilde{\pi}$ denotes the contragredient representation of $\pi$.

The logarithmic derivative of $L\left(s, \pi \times \widetilde{\pi}^{\prime}\right)$, for Res $>1$, can be written as the absolutely convergent series

$$
-\frac{L^{\prime}}{L}\left(s, \pi \times \tilde{\pi}^{\prime}\right)=-\sum_{n=1}^{\infty} \frac{\Lambda(n) \alpha_{\pi \times \tilde{\pi}^{\prime}}(n)}{n^{s}}=-\sum_{n=1}^{\infty} \frac{\Lambda_{\pi, \pi^{\prime}}(n)}{n^{s}},
$$

where

$$
\alpha_{\pi \times \widetilde{\pi}^{\prime}}\left(p^{r f_{p}}\right)=\sum_{v \mid p} f_{p}\left(\sum_{j=1}^{m} \alpha_{\pi}(v, j)^{r}\right)\left(\sum_{k=1}^{m^{\prime}}{\overline{\alpha_{\pi^{\prime}}(v, k)}}^{r}\right),
$$

and $\alpha_{\pi \times \widetilde{\pi}^{\prime}}\left(p^{r}\right)=0$ if $f_{p} \nmid r$.

Similarly, at the infinite place $v \in S_{\infty}$, the archimedean local factor $L_{v}\left(s, \pi_{v} \times \widetilde{\pi}_{v}^{\prime}\right)$ can be written as a product

$$
L_{v}\left(s, \pi_{v} \times \widetilde{\pi}_{v}^{\prime}\right)=\prod_{j=1}^{m} \prod_{k=1}^{m^{\prime}} \Gamma_{v}\left(s+\mu_{\pi \times \widetilde{\pi}^{\prime}}(v, j, k)\right),
$$

where $\mu_{\pi \times \widetilde{\pi}^{\prime}}(v, j, k)=\mu_{\pi}(v, j)+\overline{\mu_{\pi^{\prime}}(v, k)}$, at the infinite places $v$ unramified for both $\pi$ and $\pi^{\prime}$. In the case when infinite place $v$ is ramified for $\pi$ or $\pi^{\prime}$, parameters $\mu_{\pi \times \widetilde{\pi}^{\prime}}(v, j, k)$ are described in [28, Appendix], where it is also proved that $\mu_{\pi \times \widetilde{\pi}^{\prime}}(v, j, k), j=1, \ldots, m, k=1, \ldots, m^{\prime}$ satisfy the trivial bound $\operatorname{Re} \mu_{\pi \times \widetilde{\pi}^{\prime}}(v, j, k)>-1$.

As proved in $[9,15,16,24,32-35]$ (see also [6, Th. 9.1. and Th. 9.2.]), the completed Rankin-Selberg $L$-function

$$
\Lambda\left(s, \pi \times \widetilde{\pi}^{\prime}\right)=L\left(s, \pi \times \widetilde{\pi}^{\prime}\right) \prod_{v \in S_{\infty}} L_{v}\left(s, \pi_{v} \times \widetilde{\pi}_{v}^{\prime}\right)
$$

extends to a meromorphic function of order 1 on the whole complex plane, bounded (away from its possible poles) in vertical strips. It has simple poles at $s=1+i t_{0}$ and $s=i t_{0}$, arising from $L\left(s, \pi \times \widetilde{\pi}^{\prime}\right)$ if and only if $m=m^{\prime}$ and $\pi^{\prime} \cong \pi \otimes|\operatorname{det}|^{i t_{0}}$, for some $t_{0} \in \mathbb{R}$. Otherwise, it is an entire function. 
The functional equation satisfied by the Rankin-Selberg $L$-function can be written as

$$
L\left(s, \pi \times \widetilde{\pi}^{\prime}\right) \Psi_{\pi, \pi^{\prime}}(s)=\bar{L}\left(1-s, \pi \times \tilde{\pi}^{\prime}\right),
$$

where

$$
\Psi_{\pi, \pi^{\prime}}(s)=\frac{Q_{\pi \times \widetilde{\pi}^{\prime}}^{s-\frac{1}{2}}}{\epsilon\left(\pi \times \widetilde{\pi}^{\prime}\right)} \prod_{v \in S_{\infty}} \prod_{j=1}^{m} \prod_{k=1}^{m^{\prime}} \frac{\Gamma_{v}\left(s+\mu_{\pi \times \widetilde{\pi}^{\prime}}(v, j, k)\right)}{\Gamma_{v}\left(1-s+\overline{\mu_{\pi \times \widetilde{\pi}^{\prime}}(v, j, k)}\right)},
$$

$Q_{\pi \times \widetilde{\pi}^{\prime}}>0$ is the arithmetic conductor, $\epsilon\left(\pi \times \tilde{\pi}^{\prime}\right)$ is a complex number of modulus 1 and $\bar{L}\left(s, \pi \times \tilde{\pi}^{\prime}\right)=\overline{L\left(\bar{s}, \pi \times \tilde{\pi}^{\prime}\right)}$.

The zeros of $\Lambda\left(s, \pi \times \widetilde{\pi}^{\prime}\right)$ are called the non-trivial zeros of $L\left(s, \pi \times \tilde{\pi}^{\prime}\right)$. They lie in the strip $0<\operatorname{Re} s<1$, see [31]. Function $L\left(s, \pi \times \tilde{\pi}^{\prime}\right)$ may also have trivial zeros, which arise from the poles of the local $L$-factors at infinite places. There are finitely many of them inside the critical strip $0 \leq \operatorname{Re} s \leq 1$, at points $s=-\mu_{\pi \times \widetilde{\pi}^{\prime}}(v, j, k)$, for those $v \in S_{\infty}, j \in\{1, \ldots, m\}$ and $k \in$ $\left\{1, \ldots, m^{\prime}\right\}$ such that $\operatorname{Re} \mu_{\pi \times \widetilde{\pi}^{\prime}}(v, j, k) \leq 0$.

Additionally, if at least one of representations $\pi$ and $\pi^{\prime}$ is self-contragredient, function $L\left(s, \pi \times \widetilde{\pi}^{\prime}\right)$ possesses a Landau-type zero-free region

$$
\operatorname{Re} s>1-\frac{C}{\log \left(Q_{\pi} Q_{\pi^{\prime}}(|t|+2)\right)},|t| \geq 1,
$$

where $C$ is an explicit constant depending only on $m$ and $m^{\prime}$ and $Q_{\pi}, Q_{\pi^{\prime}}$ are analytic conductors of representations $\pi$ and $\pi^{\prime}$, respectively (see $[8,25,29]$ ).

2.2. The Prime Number Theorem for the Rankin-Selberg L-function. The analogue of the Prime Number Theorem for the Rankin-Selberg $L$-function is a theorem which describes the asymptotic behavior of the sum

$$
\psi_{\pi, \pi^{\prime}}(x)=\sum_{n \leq x} \Lambda(n) \alpha_{\pi \times \widetilde{\pi}^{\prime}}(n)=\sum_{n \leq x} \Lambda_{\pi, \pi^{\prime}}(n)
$$

as $x \rightarrow \infty$. It is the following theorem.

Theorem 2.1 ([10]). Let $E$ be a Galois extension of $\mathbb{Q}$ of degree l. Let $\pi$ and $\pi^{\prime}$ be irreducible cuspidal unitary representations of $G L_{m}\left(E_{\mathbb{A}}\right)$ and $G L_{m^{\prime}}\left(E_{\mathbb{A}}\right)$, respectively. Then,

$$
\psi_{\pi, \pi^{\prime}}(x)=\delta_{\pi, \pi^{\prime}}\left(t_{0}\right) \frac{x^{1+i t_{0}}}{1+i t_{0}}+g_{\pi, \pi^{\prime}}(x),
$$

where

$$
\delta_{\pi, \pi^{\prime}}\left(t_{0}\right)=\left\{\begin{array}{l}
1, \quad \text { if } m=m^{\prime} \text { and } \pi^{\prime} \cong \pi \otimes|\operatorname{det}|^{i t_{0}}, \text { for some } t_{0} \in \mathbb{R} \\
0, \quad \text { otherwise, }
\end{array}\right.
$$

and $g_{\pi, \pi^{\prime}}(x)=o(x)$, as $x \rightarrow \infty$, if $\pi^{\prime} \cong \pi$ and $g_{\pi, \pi^{\prime}}(x)=O(x)$, as $x \rightarrow \infty$, otherwise. If we additionally assume that at least one of representations $\pi$ 
or $\pi^{\prime}$ is self-contragredient, then $g_{\pi, \pi^{\prime}}(x)=O(x \exp (-c \sqrt{\log x}))$, for some constant $c>0$, as $x \rightarrow \infty$.

Proof. If $\pi^{\prime} \cong \pi$, then $\psi_{\pi, \pi^{\prime}} \sim x$, by [10, Lemma 3.3.]. Application of the Hölder inequality yields that $\left|\psi_{\pi, \pi^{\prime}}(x)\right|^{2} \leq\left|\psi_{\pi, \pi}(x)\right|\left|\psi_{\pi^{\prime}, \pi^{\prime}}(x)\right| \leq x^{2}$, hence $g_{\pi, \pi^{\prime}}(x)=O(x)$, as $x \rightarrow \infty$. If one of representations $\pi$ and $\pi^{\prime}$ is self-contragredient the statement of theorem is proved in [10] by generalizing result of Liu and Ye ([21]).

2.3. The Selberg orthogonality conjecture and Hypothesis H. The Selberg orthogonality conjecture for automorphic $L$-functions on $G L_{m}\left(\mathbb{Q}_{\mathbb{A}}\right)$ was posed by A. Selberg in [30]. It is the following conjecture.

The Selberg orthogonality conjecture for automorphic L-functions. For any two automorphic irreducible cuspidal representations $\pi$ and $\pi^{\prime}$ of $G L_{m}\left(\mathbb{Q}_{\mathbb{A}}\right)$ and $G L_{m^{\prime}}\left(\mathbb{Q}_{\mathbb{A}}\right)$

$$
\sum_{p \leq x} \frac{\alpha_{\pi}(p) \bar{\alpha}_{\pi^{\prime}}(p)}{p}=\delta_{\pi, \pi^{\prime}}(0) \log \log x+O(1), \text { as } x \rightarrow \infty .
$$

Z. Rudnick and P. Sarnak ([28]) have proved the Selberg orthogonality conjecture in the case when $\pi$ and $\pi^{\prime}$ are equivalent, under the following hypothesis on convergence of series of prime powers.

Hypothesis $H$. For any automorphic irreducible cuspidal representation $\pi$ of $G L_{m}\left(\mathbb{Q}_{\mathbb{A}}\right)$ and any fixed $k \geq 2$

$$
\sum_{p} \frac{\left|\alpha_{\pi}\left(p^{k}\right)\right|^{2} \log ^{2} p}{p^{k}}<\infty .
$$

The Hypothesis $\mathrm{H}$ was posed in order to deduce the behavior of a certain sum over primes arising in the proof of the Selberg orthogonality conjecture from the asymptotic formula for the sum over all integers.

The Hypothesis $\mathrm{H}$ was generalized to the setting of Galois extensions $E$ of degree $l$ over $\mathbb{Q}$ by J. Liu and Y. Ye in [22]. We will refer to this hypothesis as Hypothesis $\mathrm{H}$ over $E$.

Hypothesis $H$ over E. For any automorphic irreducible cuspidal representation $\pi$ of $G L_{m}\left(E_{\mathbb{A}}\right)$ with unitary central character and any fixed $k \geq 2$

$$
\sum_{p} \frac{\log ^{2} p}{p^{k f_{p}}} \sum_{v \mid p}\left|\sum_{j=1}^{m} \alpha_{\pi}^{k}(v, j)\right|^{2}<\infty .
$$

Hypothesis $\mathrm{H}$ over $E$ is trivial when $m=1$, for $m=2$ it is a consequence of the bound $\left|\alpha_{\pi}(v, j)\right| \leq p^{f_{p} / 9}$ proved in [18]. When $m=3$, Hypothesis $\mathrm{H}$ over $E$ is proved in [22, Appendix], and in the case $m=4$, it is a consequence of [17], as pointed out by Kim and Sarnak in [17, Appendix]. An immediate consequence of equation (2.5) and the definition of $\Lambda_{\pi, \pi^{\prime}}(n)$ is the following lemma. 
Lemma 2.2. Let $E$ be Galois extension of $\mathbb{Q}$ of finite degree $l$ and let $\pi$ and $\pi^{\prime}$ be two irreducible automorphic unitary cuspidal representations of $G L_{m}\left(E_{\mathbb{A}}\right)$ and $G L_{m^{\prime}}\left(E_{\mathbb{A}}\right)$, respectively. Then, for $m, m^{\prime} \leq 4$ or under Hypothesis $H$ over $E$ if $m>4$ or $m^{\prime}>4$

$$
\sum_{n \leq x} \frac{\Lambda_{\pi, \pi^{\prime}}(n)}{n}=\sum_{p \leq x} \frac{f_{p} \log p}{p^{f_{p}}} \sum_{v \mid p}\left(\sum_{j=1}^{m} \alpha_{\pi}(v, j)\right)\left(\sum_{j=1}^{m^{\prime}} \overline{\alpha_{\pi^{\prime}}(v, j)}\right)+O(1),
$$

as $x \rightarrow \infty$.

2.4. The Li coefficients for the Rankin-Selberg L-function. Generalized Li coefficients for the Rankin-Selberg $L$-function $L\left(s, \pi \times \widetilde{\pi}^{\prime}\right)$ are defined as

$$
\lambda_{\pi, \pi^{\prime}}(n)=\sum_{\rho \neq 0}^{*}\left(1-\left(1-\frac{1}{\rho}\right)^{n}\right),
$$

where the sum is taken over all non-trivial zeros of the function $L\left(s, \pi \times \tilde{\pi}^{\prime}\right)$ and $*$ means that the above sum is taken in the sense of the limit $\lim _{T \rightarrow \infty} \sum_{|\operatorname{Im} \rho| \leq T}$.

In a general case of a number field $E$ of degree $l=[E: \mathbb{Q}]$, it is proved in [27] that coefficients $\lambda_{\pi, \pi^{\prime}}(n)$ are well defined for all integers $n$ and that the generalized Riemann hypothesis for the Rankin-Selberg $L$-function $L(s, \pi \times$ $\left.\widetilde{\pi}^{\prime}\right)$ is equivalent to non-negativity of numbers $\operatorname{Re} \lambda_{\pi, \pi^{\prime}}(n)$, for all $n \in \mathbb{N}$. Furthermore, an arithmetic expression for $\lambda_{\pi, \pi^{\prime}}(n)$ is obtained, as stated in the following theorem.

THEOREM 2.3 ([27]). Let $\pi$ and $\pi^{\prime}$ be two automorphic unitary cuspidal representations of $G L_{m}\left(E_{\mathbb{A}}\right)$ and $G L_{m^{\prime}}\left(E_{\mathbb{A}}\right)$, respectively. Then, for all $n \in \mathbb{N}$ and $t_{0} \in \mathbb{R} \backslash\{0\}$

$$
\lambda_{\pi, \pi^{\prime}}(-n)=\sum_{j=1}^{n}\left(\begin{array}{l}
n \\
j
\end{array}\right) \gamma_{\pi, \pi^{\prime}}(j-1)+D_{\pi, \pi^{\prime}}\left(t_{0}\right)+S_{\infty}\left(n, \pi, \pi^{\prime}\right),
$$

where

$$
D_{\pi, \pi^{\prime}}\left(t_{0}\right)=\delta_{\pi, \pi^{\prime}}\left(t_{0}\right)\left(2-\left(1+\frac{1}{i t_{0}}\right)^{n}-\left(1-\frac{1}{1-i t_{0}}\right)^{n}\right)
$$

and $S_{\infty}\left(n, \pi, \pi^{\prime}\right)$ denotes the archimedean contribution to the generalized Li coefficient given by

$$
\begin{aligned}
S_{\infty}\left(n, \pi, \pi^{\prime}\right) & =\delta_{\pi, \pi^{\prime}}(0)+\frac{n}{2}\left(\log Q_{\pi \times \widetilde{\pi}^{\prime}}-l m m^{\prime} \log \pi\right) \\
& -l m m^{\prime}+\sum_{i=1}^{l m m^{\prime}}\left(\frac{\mu_{\pi \times \widetilde{\pi}^{\prime}}(i)}{1+\mu_{\pi \times \widetilde{\pi}^{\prime}}(i)}\right)^{n}+\sum_{j=1}^{n}\left(\begin{array}{l}
n \\
j
\end{array}\right) \eta_{\pi, \pi^{\prime}}(j-1),
\end{aligned}
$$


where

$$
\eta_{\pi, \pi^{\prime}}(0)=\frac{1}{2} \sum_{i=1}^{l m m^{\prime}} \frac{\Gamma^{\prime}}{\Gamma}\left(\frac{3+\mu_{\pi \times \widetilde{\pi}^{\prime}}(i)}{2}\right)
$$

and

$$
\eta_{\pi, \pi^{\prime}}(j-1)=\frac{(-1)^{j}}{2^{j}} \sum_{i=1}^{l m m^{\prime}} \sum_{t=0}^{\infty}\left(t+\frac{3+\mu_{\pi \times \widetilde{\pi}^{\prime}}(i)}{2}\right)^{-j}, \text { for } j \geq 2 .
$$

Here, $r_{1}$ is the number of real places, $r_{2}$ is the number of complex places and we put $\mu_{\pi \times \widetilde{\pi}^{\prime}}(i)=\mu_{\pi \times \widetilde{\pi}^{\prime}}(v, j, k)$ for $r_{1}+r_{2}$ places $v \in S_{\infty}$ and $\mu_{\pi \times \widetilde{\pi}^{\prime}}(i)=$ $\mu_{\pi \times \tilde{\pi}^{\prime}}(v, j, k)+1$ for the rest of $r_{2}$ places $v \in S_{\infty}\left(j=1, \ldots, m, k=1, \ldots, m^{\prime}\right)$.

An asymptotic expression for the archimedean contribution $S_{\infty}\left(n, \pi, \pi^{\prime}\right)$ to the generalized Li coefficient $\lambda_{\pi, \pi^{\prime}}(-n)$ is obtained in [27, Theorem 2], up to a term $O\left(n^{-k}\right)$, for arbitrary $k \in \mathbb{N}$, as $n \rightarrow \infty$, where it was proved that $S_{\infty}\left(n, \pi, \pi^{\prime}\right)$ grows as $\frac{1}{2} l m m^{\prime} n \log n$, as $n \rightarrow \infty$. Derivation of asymptotic behavior as $n \rightarrow \infty$ of the non-archimedean (finite) contribution

$$
S_{\mathrm{NA}}\left(n, \pi, \pi^{\prime}\right)=\sum_{j=1}^{n}\left(\begin{array}{l}
n \\
j
\end{array}\right) \gamma_{\pi, \pi^{\prime}}(j-1)+D_{\pi, \pi^{\prime}}\left(t_{0}\right)
$$

to the generalized Li coefficient $\lambda_{\pi, \pi^{\prime}}(-n)$ is an important open problem. Combining Theorem 2 and Theorem 4 from [27] it is easy to see that, under the generalized Riemann hypothesis $S_{\mathrm{NA}}\left(n, \pi, \pi^{\prime}\right)=o(n)$, as $n \rightarrow \infty$. In general, it is very difficult to control the growth of $S_{\mathrm{NA}}\left(n, \pi, \pi^{\prime}\right)$. Based on numerical computations in some special cases (see e.g. [23]) it is conjectured that the non-archimedean contribution oscillates with a small amplitude.

\section{MAIN RESUlts}

3.1. An arithmetic formula for the generalized Euler-Stieltjes constants. Our main theorem gives an arithmetic formula for computation of coefficients $\gamma_{\pi, \pi^{\prime}}(k)$ in the Laurent series expansion (1.1) of the function $\frac{L^{\prime}}{L}\left(s, \pi \times \tilde{\pi}^{\prime}\right)$ around $s=1$.

In the proof we will need following lemmas.

LEMMA 3.1. The following identity holds true

$$
\sum_{j=0}^{k}(-1)^{j+1} \frac{x^{i t_{0}}}{i t_{0}} \sum_{a=0}^{j} \frac{(-1)^{j-a}}{\left(i t_{0}\right)^{j-a}} \frac{\log ^{a} x}{a !} \frac{\log ^{k-j} x}{(k-j) !}+\frac{x^{i t_{0}}}{\left(i t_{0}\right)^{k+1}}=0,
$$

for any $x>0, t_{0} \in \mathbb{R} \backslash\{0\}$ and every non-negative integer $k$.

Proof. We compute coefficients that correspond to different powers of logarithm, that range from 0 to $k$. For $k-j+a=k$, i.e. when $a=j$, the 
sum in (3.1) reduces to zero, by binomial theorem, since

$$
\sum_{j=0}^{k}(-1)^{j+1} \frac{x^{i t_{0}}}{i t_{0}} \frac{\log ^{k} x}{j !(k-j) !}=-\frac{x^{i t_{0}}}{i t_{0}} \frac{\log ^{k} x}{k !} \sum_{j=0}^{k}(-1)^{j} \frac{k !}{j !(k-j) !}=0 .
$$

Therefore, the coefficient multiplying $\log ^{k} x$ is zero. Arguing in a similar manner, we deduce that coefficients multiplying $\log ^{k-j+a} x$ in (3.1) are equal to 0 for all $k-j+a$ ranging from 1 to $k$. In the case when $k-j+a=0$, the corresponding coefficient in (3.1) is equal to $-\frac{x^{i t_{0}}}{\left(i t_{0}\right)^{k+1}}$, which cancels with the last term on the left hand side of (3.1). The proof is complete.

Lemma 3.2. Let $E$ be Galois extension of $\mathbb{Q}$ of finite degree $l$ and let $\pi$ and $\pi^{\prime}$ be two irreducible automorphic unitary cuspidal representations of $G L_{m}\left(E_{\mathbb{A}}\right)$ and $G L_{m^{\prime}}\left(E_{\mathbb{A}}\right)$, respectively. Let $\rho$ denote the non-trivial zeros of $L\left(s, \pi \times \tilde{\pi}^{\prime}\right)$, counted according to their multiplicities. Then, for positive integers $j$ and $k$

(i)

$$
\log ^{j} x \sum_{\rho}\left|\frac{x^{\rho-1}}{(\rho-1)^{k+1}}\right|=o(1), \log ^{j} x \sum_{\rho}\left|\frac{x^{\rho-1}}{\rho(\rho-1)}\right|=o(1),
$$

as $x \rightarrow \infty$.

(ii) If, additionally at least one of representations $\pi$ and $\pi^{\prime}$ is self-contragredient, then for $T \sim \exp (\sqrt{\log x})$ such that $T$ is not an imaginary part of any non-trivial zero of $L\left(s, \pi \times \tilde{\pi}^{\prime}\right)$ we have

$$
\max \left\{\sum_{|\operatorname{Im} \rho| \leq T}\left|\frac{x^{\rho-1}}{(\rho-1)^{k+1}}\right|, \sum_{|\operatorname{Im} \rho| \leq T}\left|\frac{x^{\rho-1}}{\rho(\rho-1)}\right|\right\}=O(\exp (-C \sqrt{(\log x)})),
$$

as $x \rightarrow \infty$, for some constant $C>0$ independent of $x$.

Proof. (i) Non-vanishing of $L\left(s, \pi \times \widetilde{\pi}^{\prime}\right)$ on the line Res $=1$ implies that $\lim _{x \rightarrow \infty}\left(\log ^{j} x\right)\left|x^{\rho-1}\right|=0$, hence to prove (i) it suffices to show that we may pass to the limit inside the sums. Function $L\left(s, \pi \times \widetilde{\pi}^{\prime}\right)$ is of order one, hence the series $\sum_{\rho}(\rho-1)^{-(k+1)}$ and $\sum_{\rho}(\rho(\rho-1))^{-1}$ converge absolutely and dominate the series

$$
\sum_{\rho} \frac{\left|x^{\rho-1}\right| \log ^{j} x}{|\rho-1|^{k+1}} \text { and } \sum_{\rho} \frac{\left|x^{\rho-1}\right|}{|\rho(\rho-1)|} .
$$

Therefore, by Weierstrass theorem, the series (3.2) converge uniformly in $x$, hence we may pass to the limit as $x \rightarrow \infty$ inside the sums in (i). This completes the proof.

(ii) Additional assumption that at least one of the representations $\pi$ and $\pi^{\prime}$ is self-contagredient enables us to use Landau type zero free region and thus 
improve the error term in (i). Namely, (2.3) implies that for $T \sim \exp (\sqrt{\log x})$ and $\operatorname{Im} \rho \leq T$

$$
\left|x^{\rho-1}\right| \ll \exp \left(-C \frac{\log x}{\log T}\right) \ll \exp (-C \sqrt{\log x}),
$$

as $x \rightarrow \infty$, for some constant $C>0$ independent on $x$ and $T$. The statement now follows from the absolute convergence of the series $\sum_{\rho}(\rho-1)^{-(k+1)}$ for $k \geq 1$ and the series $\sum_{\rho}(\rho(\rho-1))^{-1}$.

Lemma 3.3. Let $E$ be Galois extension of $\mathbb{Q}$ of finite degree $l$ and let $\pi$ and $\pi^{\prime}$ be two irreducible automorphic unitary cuspidal representations of $G L_{m}\left(E_{\mathbb{A}}\right)$ and $G L_{m^{\prime}}\left(E_{\mathbb{A}}\right)$, respectively. Let $k$ be a positive integer.

(i) Let $\eta$ denote trivial zeros of $L\left(s, \pi \times \widetilde{\pi}^{\prime}\right)$, counted according to their multiplicities. Then

$$
\sum_{0<\operatorname{Re} \eta<1} \frac{x^{\eta-1}}{(\eta-1)^{k+1}}=O\left(x^{-\delta}\right), \sum_{0<\operatorname{Re} \eta<1} \frac{x^{\eta-1}}{\eta(\eta-1)}=O\left(x^{-\delta}\right),
$$

for some constant $\delta \in(0,1)$, as $x \rightarrow \infty$.

(ii) For any $\epsilon>0$ and $T>1$

$$
\int_{-1-\epsilon-i T}^{-1-\epsilon+i T}\left(-\frac{L^{\prime}}{L}\left(s+1, \pi \times \widetilde{\pi}^{\prime}\right) \frac{x^{s}}{s^{k+1}}\right) d s=O\left(x^{-1-\epsilon}\right),
$$

as $x \rightarrow \infty$.

Proof. Since representations $\pi$ and $\pi^{\prime}$ are arbitrarily chosen, but fixed in the sequel we denote the function $L\left(s, \pi \times \widetilde{\pi}^{\prime}\right)$ simply by $L(s)$.

(i) The trivial zeros $\eta$ of $L(s)$ such that $0<\operatorname{Re} \eta<1$ are located at points $\eta=-\mu_{\pi \times \widetilde{\pi}^{\prime}}(v, j, k)$, for $\mu_{\pi \times \widetilde{\pi}^{\prime}}(v, j, k)$ such that $\operatorname{Re} \mu_{\pi \times \widetilde{\pi}^{\prime}}(v, j, k) \leq 0$. The inequality $\operatorname{Re} \mu_{\pi \times \widetilde{\pi}^{\prime}}(v, j, k)>-1$ implies that $\operatorname{Re} \eta<1$, hence, there exists $\delta>0$, such that $\operatorname{Re}(\eta-1) \leq-\delta<0$. This completes the proof, since the sums over trivial zeros are finite.

(ii) The functional equation $(2.2)$ and the fact that $\frac{\Gamma^{\prime}}{\Gamma}(s)=O(\log |s|)$, as $|s| \rightarrow \infty$ yields

$$
-\frac{L^{\prime}}{L}(-\epsilon+i t)=O(\log t)+O(1)=O(\log t)
$$


as $t \rightarrow \infty$. Therefore, for $k \geq 1$, one has

$$
\begin{aligned}
\int_{-1-\epsilon-i T}^{-1-\epsilon+i T}\left(-\frac{L^{\prime}}{L}(s+1) \frac{x^{s}}{s^{k+1}}\right) d s & \ll \int_{-T}^{T}\left|-\frac{L^{\prime}}{L}(-\epsilon+i t)\right| \frac{x^{-1-\epsilon}}{(-1-\epsilon+i t)^{k+1}} d t \\
& \ll x^{-1-\epsilon} \int_{1}^{T} \frac{\log t}{t^{2}} d t=O\left(x^{-1-\epsilon}\right),
\end{aligned}
$$

as $x \rightarrow \infty$. The proof is complete.

TheOREm 3.4. Let $E$ be Galois extension of $\mathbb{Q}$ of finite degree $l$ and let $\pi$ and $\pi^{\prime}$ be two irreducible automorphic unitary cuspidal representations of $G L_{m}\left(E_{\mathbb{A}}\right)$ and $G L_{m^{\prime}}\left(E_{\mathbb{A}}\right)$, respectively and such that at least one of $\pi$ or $\pi^{\prime}$ is self-contragredient. Then for all integers $k \geq 0$

$$
\begin{aligned}
\gamma_{\pi, \pi^{\prime}}(k)= & \frac{(-1)^{k+1}}{k !}\left(\sum_{n \leq x} \frac{\Lambda_{\pi, \pi^{\prime}}(n)}{n} \log ^{k} n-\delta_{\pi, \pi^{\prime}}(0) \frac{\log ^{k+1} x}{k+1}\right. \\
& \left.-\delta_{\pi, \pi^{\prime}}\left(t_{0}\right) A_{\pi, \pi^{\prime}, k}\left(t_{0}, x\right)\right)+O\left(\log ^{k} x \exp (-C \sqrt{\log x})\right)
\end{aligned}
$$

for some $C>0$, as $x \rightarrow \infty$, where $t_{0} \in \mathbb{R} \backslash\{0\}$ and

$$
A_{\pi, \pi^{\prime}, k}\left(t_{0}, x\right)=k !\left(\frac{x^{i t_{0}}}{i t_{0}} \sum_{j=0}^{k} \frac{(-1)^{k-j}}{\left(i t_{0}\right)^{k-j}} \frac{\log ^{j} x}{j !}\right) .
$$

Proof. We denote the function $L\left(s, \pi \times \widetilde{\pi}^{\prime}\right)$ by $L(s)$ and use the induction in $k$.

In the special case when representations $\pi$ and $\pi^{\prime}$ are attached to $\mathrm{GL}_{m}\left(\mathbb{Q}_{A}\right)$ and $\mathrm{GL}_{m^{\prime}}\left(\mathbb{Q}_{A}\right)$, respectively, formula (3.3) with $k=0$ is obtained in [2, Proposition 4.1.]. The proof of (3.3) when $k=0$, given in [2] is based on evaluation of the integral

$$
\frac{1}{2 \pi i} \int_{c-i T}^{c+i T} \frac{L^{\prime}}{L}(s+1) \frac{x^{s}}{s(s+1)} d s
$$

for some positive constant $c$ and $T>1$ in two different ways combined with the properties of the $L$-function appearing in the integrand. The $L$ function attached to irreducible automorphic unitary cuspidal representations of $G L_{m}\left(E_{\mathbb{A}}\right)$ and $G L_{m^{\prime}}\left(E_{\mathbb{A}}\right)$ possesses all properties needed in the proof of 
[2, Theorem 2.1.], hence, repeating the steps of the proof we get

$$
\begin{aligned}
\sum_{n \leq x}\left(1-\frac{n}{x}\right) \frac{\Lambda_{\pi, \pi^{\prime}}(n)}{n}= & \delta_{\pi, \pi^{\prime}}(0)(\log x-1)-\gamma_{\pi, \pi^{\prime}}(0) \\
& +\delta_{\pi, \pi^{\prime}}\left(t_{0}\right) \frac{x^{i t_{0}}}{i t_{0}\left(1+i t_{0}\right)}+\sum_{|\operatorname{Im} \rho| \leq T} \frac{x^{\rho-1}}{\rho(1-\rho)} \\
& +\sum_{0<\operatorname{Re} \eta<1} \frac{x^{\eta-1}}{\eta(1-\eta)}+O\left(\max \left\{\frac{\log x}{x}, \frac{x^{c}}{T}\right\}\right),
\end{aligned}
$$

as $T, x \rightarrow \infty$, where $c>0$ is arbitrary. Here, $\rho$ denotes non-trivial zeros of $L$ and $\eta$ denotes trivial zeros of $L$, all counted according to their multiplicities. Application of the formula (2.4) and Lemma 3.3 (i) yields that

$$
\begin{aligned}
& O\left(\max \left\{x^{-\delta}, \frac{x^{c}}{T}, \frac{g_{\pi, \pi^{\prime}}(x)}{x}, \sum_{|\operatorname{Im} \rho| \leq T}\left|\frac{x^{\rho-1}}{\rho(1-\rho)}\right|\right\}\right) \\
& =\sum_{n \leq x} \frac{\Lambda_{\pi, \pi^{\prime}}(n)}{n}-\delta_{\pi, \pi^{\prime}}(0) \log x+\gamma_{\pi, \pi^{\prime}}(0)-\delta_{\pi, \pi^{\prime}}\left(t_{0}\right) \frac{x^{i t_{0}}}{i t_{0}}
\end{aligned}
$$

as $x \rightarrow \infty$. Formula (3.5) holds true for representations $\pi$ and $\pi^{\prime}$ that are not necessarily self-contragredient, since it is obtained by repeating the arguments of $\left[2\right.$, Theorem 2.1.] and using estimates that hold true for general $\pi$ and $\pi^{\prime}$.

Taking $c=1 / \log x, T \sim \exp (\sqrt{\log x})$, applying Lemma 3.2 (ii) and Theorem 2.1 to $(3.5)$ we deduce that

$O(\exp (-C \sqrt{\log x}))=\sum_{n \leq x} \frac{\Lambda_{\pi, \pi^{\prime}}(n)}{n}-\delta_{\pi, \pi^{\prime}}(0) \log x+\gamma_{\pi, \pi^{\prime}}(0)-\delta_{\pi, \pi^{\prime}}\left(t_{0}\right) \frac{x^{i t_{0}}}{i t_{0}}$,

as $x \rightarrow 0$, for some $C>0$, which is exactly formula (3.3), for $k=0$.

Let us now assume that $k \geq 1$. We start with the integral

$$
\frac{1}{2 \pi i} \int_{c-i \infty}^{c+i \infty}\left(-\frac{L^{\prime}}{L}(s+1) \frac{x^{s}}{s^{k+1}}\right) d s
$$

that we evaluate in two different ways. Here, $c$ is a positive real number and $x>1$ is not an integer. 
First, using series representation (2.1), integration by parts and integral given in $[37$, p. 107] we write $(3.7)$ as

$$
\begin{gathered}
\frac{1}{2 \pi i} \int_{c-i \infty}^{c+i \infty}\left(-\frac{L^{\prime}}{L}(s+1) \frac{x^{s}}{s^{k+1}}\right) d s=\sum_{n \leq x} \frac{\Lambda_{\pi, \pi^{\prime}}(n)}{n} \frac{1}{k !}\left(\log \frac{x}{n}\right)^{k} \\
=\sum_{j=0}^{k} \frac{(-1)^{j}}{j !(k-j) !}\left(\sum_{n \leq x} \frac{\Lambda_{\pi, \pi^{\prime}}(n)}{n} \log ^{j} n\right) \log ^{k-j} x .
\end{gathered}
$$

On the other hand, integral (3.7) can be evaluated using contour integration. Namely,

$$
\frac{1}{2 \pi i} \int_{c-i \infty}^{c+i \infty}\left(-\frac{L^{\prime}}{L}(s+1) \frac{x^{s}}{s^{k+1}}\right) d s=\frac{1}{2 \pi i} \int_{c-i T}^{c+i T}\left(-\frac{L^{\prime}}{L}(s+1) \frac{x^{s}}{s^{k+1}}\right) d s+O\left(\frac{x^{c}}{T^{k}}\right),
$$

as $x, T \rightarrow \infty$. The last integral is computed by integration along the boundaries of the rectangle $R_{c, T, \epsilon}$ with vertices $c-i T, c+i T,-1-\epsilon+i T,-1-\epsilon-i T$, where $c, \epsilon>0$ and $T>1$ are chosen such that $L(s+1)$ has no zeros on the boundaries and such that trivial zeros $\eta$ of the function $L(s)$ such that $0<\operatorname{Re} \eta<1$ are all trivial zeros of $L$ inside the rectangle $R_{c, T, \epsilon}$.

Let $\rho$ and $\eta$ denote non-trivial and trivial zeros of $L(s)$, such that $|\operatorname{Im} \rho| \leq$ $T$ and $0<\operatorname{Re} \eta<1$, respectively. Then, $(\rho-1)$ and $(\eta-1)$ are poles, counted according to their multiplicities, of the integrand in (3.9) inside the rectangle $R_{c, T, \epsilon}$.

If $\pi^{\prime} \cong \pi$, then $s=0$ is a pole of order $k+2$ and $s=-1$ is a simple pole of the integrand in (3.9). If $\pi^{\prime} \cong \pi \otimes|\operatorname{det}|^{i t_{0}}$, for some $t_{0} \in \mathbb{R} \backslash\{0\}$, then $s=0$ is pole of order $k+1$, while $s=i t_{0}$ and $s=-1+i t_{0}$ are simple poles. When $\pi^{\prime} ¥ \pi \otimes|\operatorname{det}|^{i t_{0}}, t_{0} \in \mathbb{R}$, point $s=0$ is pole of order $k+1$ of the integrand in (3.9).

If $s=0$ is a pole of order $k+1$, its residue is easily found from the series representation

$$
\begin{aligned}
-\frac{L^{\prime}}{L}(s+1) \frac{x^{s}}{s^{k+1}} & =-\left(\sum_{l=0}^{\infty} \gamma_{\pi, \pi^{\prime}}(l) s^{l}\right)\left(\sum_{m=0}^{\infty} \frac{\log ^{m} x}{m !} s^{m}\right) s^{-k-1} \\
& =-\sum_{j=0}^{\infty} \sum_{l=0}^{j} \gamma_{\pi, \pi^{\prime}}(l) \frac{\log ^{j-l} x}{(j-l) !} s^{j-k-1}
\end{aligned}
$$

and thus

$$
\operatorname{Res}_{s=0}\left(-\frac{L^{\prime}}{L}(s+1) \frac{x^{s}}{s^{k+1}}\right)=-\sum_{l=0}^{k} \gamma_{\pi, \pi^{\prime}}(l) \frac{\log ^{k-l} x}{(k-l) !} .
$$


In the case when $s=0$ is a pole of order $k+2$, i.e. in the case when $\pi^{\prime} \cong \pi$, there is an additional term in the residue that comes from the fact that in this case

$$
\frac{L^{\prime}}{L}(s+1)=\frac{-1}{s}+\sum_{l=0}^{\infty} \gamma_{\pi, \pi^{\prime}}(l) s^{l},
$$

therefore

$$
\operatorname{Res}_{s=0}\left(-\frac{L^{\prime}}{L}(s+1) \frac{x^{s}}{s^{k+1}}\right)=-\sum_{l=0}^{k} \gamma_{\pi, \pi^{\prime}}(l) \frac{\log ^{k-l} x}{(k-l) !}+\frac{\log ^{k+1} x}{(k+1) !} .
$$

Application of the argument principle now yields

$$
\begin{aligned}
& -\frac{1}{2 \pi i} \int_{R_{c, T, \epsilon}} \frac{L^{\prime}}{L}(s+1) \frac{x^{s}}{s^{k+1}} d s \\
& =-\sum_{|\operatorname{Im} \rho| \leq T} \frac{x^{\rho-1}}{(\rho-1)^{k+1}}-\sum_{0<\operatorname{Re} \eta<1} \frac{x^{\eta-1}}{(\eta-1)^{k+1}}-\sum_{j=0}^{k} \gamma_{\pi, \pi^{\prime}}(j) \frac{\log ^{k-j} x}{(k-j) !} \\
& \quad+\delta_{\pi, \pi^{\prime}}(0)\left(\frac{\log ^{k+1} x}{(k+1) !}-\frac{(-1)^{k}}{x}\right) \\
& \quad+\delta_{\pi, \pi^{\prime}}\left(t_{0}\right)\left(\frac{x^{i t_{0}}}{\left(i t_{0}\right)^{k+1}}+\frac{x^{-1+i t_{0}}}{\left(-1+i t_{0}\right)^{k+1}}\right) .
\end{aligned}
$$

It is easy to obtain that integrals over horizontal lines of the rectangle $R_{c, T, \epsilon}$ are $O\left(x^{c} / T\right)$, as $x, T \rightarrow \infty$, hence Lemma 3.3, together with (3.10) and (3.9) yields that

$$
\begin{aligned}
& \frac{1}{2 \pi i} \int_{c-i \infty}^{c+i \infty}\left(-\frac{L^{\prime}}{L}(s+1) \frac{x^{s}}{s^{k+1}}\right) d s \\
& =-\sum_{j=0}^{k} \gamma_{\pi, \pi^{\prime}}(j) \frac{\log ^{k-j} x}{(k-j) !}+\delta_{\pi, \pi^{\prime}}(0) \frac{\log ^{k+1} x}{(k+1) !}+\delta_{\pi, \pi^{\prime}}\left(t_{0}\right) \frac{x^{i t_{0}}}{\left(i t_{0}\right)^{k+1}} \\
& \quad+O\left(\max \left\{\frac{x^{c}}{T}, x^{-\delta}, \sum_{|\operatorname{Im} \rho| \leq T}\left|\frac{x^{\rho-1}}{(\rho-1)^{k+1}}\right|\right\}\right)
\end{aligned}
$$

as $x, T \rightarrow \infty$. Equation (3.11) holds true for $\pi$ and $\pi^{\prime}$ that are not necessarily self-contragredient.

Taking $c=1 / \log x$ and $T \sim \exp (\sqrt{\log x})$ and applying Lemma 3.2 (ii) to (3.11) yields that there exists unique constant $C$ independent of $x$ and such that the error term on the right hand side of $(3.11)$ is $O(\exp (-C \sqrt{\log x}))$. 
Now, comparing (3.11) and (3.8) we get, for $k \geq 1$

$$
\begin{gathered}
O(\exp (-C \sqrt{\log x}))=-\delta_{\pi, \pi^{\prime}}(0) \frac{\log ^{k+1} x}{(k+1) !}-\delta_{\pi, \pi^{\prime}}\left(t_{0}\right) \frac{x^{i t_{0}}}{\left(i t_{0}\right)^{k+1}} \\
\quad+\sum_{j=0}^{k} \frac{\log ^{k-j} x}{(k-j) !}\left(\gamma_{\pi, \pi^{\prime}}(j)+\frac{(-1)^{j}}{j !} \sum_{n \leq x} \frac{\Lambda_{\pi, \pi^{\prime}}(n)}{n} \log ^{j} n\right),
\end{gathered}
$$

as $x \rightarrow \infty$. Notice that the above formula reduces to relation (3.6) when $k=0$. Therefore, (3.12) holds true for all integers $k \geq 0$.

Now, we proceed inductively in $k$. For $k=0$ formula (3.6) is equivalent to (3.3), hence the statement of theorem holds true for $k=0$. Let $k \geq 1$ and assume that $\gamma_{\pi, \pi^{\prime}}(j)$ for all $j=0, \ldots, k-1$ are given by (3.3), as $x \rightarrow \infty$. Inserting (3.3) for $j=0, \ldots, k-1$ into (3.12) we get

$$
\begin{aligned}
& O\left(\log ^{k} x \exp (-C \sqrt{\log x})\right)=\gamma_{\pi, \pi^{\prime}}(k)+\frac{(-1)^{k}}{k !} \sum_{n \leq x} \frac{\Lambda_{\pi, \pi^{\prime}}(n)}{n} \log ^{k} n \\
& +\delta_{\pi, \pi^{\prime}}(0)\left(\sum_{j=0}^{k-1} \frac{\log ^{k-j} x}{(k-j) !} \frac{(-1)^{j} \log ^{j+1} x}{(j+1) !}-\frac{\log ^{k+1} x}{(k+1) !}\right) \\
& \quad+\delta_{\pi, \pi^{\prime}}\left(t_{0}\right)\left(\sum_{j=0}^{k-1} \frac{\log ^{k-j} x}{(k-j) !}(-1)^{j} \frac{x^{i t_{0}}}{i t_{0}} \sum_{l=0}^{j} \frac{(-1)^{j-l} \log ^{l} x}{\left(i t_{0}\right)^{j-l} l !}-\frac{x^{i t_{0}}}{\left(i t_{0}\right)^{k+1}}\right),
\end{aligned}
$$

as $x \rightarrow \infty$. Expressions multiplying $\delta_{\pi, \pi^{\prime}}(0)$ and $\delta_{\pi, \pi^{\prime}}\left(t_{0}\right)$ can be considerably simplified. Namely, since

$$
\frac{1}{(k+1) !}=\sum_{j=0}^{k} \frac{(-1)^{j}}{(j+1) !(k-j) !}
$$

we deduce that

$$
\sum_{j=0}^{k-1} \frac{\log ^{k-j} x}{(k-j) !} \frac{(-1)^{j} \log ^{j+1} x}{(j+1) !}-\frac{\log ^{k+1} x}{(k+1) !}=\frac{(-1)^{k+1} \log ^{k+1} x}{(k+1) !},
$$

while Lemma 3.1 implies that

$$
\sum_{j=0}^{k-1} \frac{\log ^{k-j} x}{(k-j) !}(-1)^{j} \frac{x^{i t_{0}}}{i t_{0}} \sum_{l=0}^{j} \frac{(-1)^{j-l} \log ^{l} x}{\left(i t_{0}\right)^{j-l} l !}-\frac{x^{i t_{0}}}{\left(i t_{0}\right)^{k+1}}=\frac{(-1)^{k+1}}{k !} A_{\pi, \pi^{\prime}, k}\left(t_{0}, x\right) .
$$

Therefore, formula (3.13) reduces to (3.3), thus, we conclude that (3.3) holds true for all integers $k \geq 0$. The proof is completed.

Inserting formula (3.3) into the formula for the non-archimedean contribution to the $n$th generalized Li coefficient immediately yields the following corollary. 
COROLlaRY 3.5. Under assumptions of Theorem 3.4 the non-archimedean contribution (2.7) to the generalized Li coefficient possesses the following, arithmetic representation

$$
\begin{aligned}
S_{\mathrm{NA}}=\sum_{k=1}^{n}\left(\begin{array}{l}
n \\
k
\end{array}\right) \frac{(-1)^{k}}{(k-1) !}\left(\sum_{n \leq x} \frac{\Lambda_{\pi, \pi^{\prime}}(n)}{n} \log ^{k-1} n-\delta_{\pi, \pi^{\prime}}(0) \frac{\log ^{k} x}{k}\right. \\
\left.-\delta_{\pi, \pi^{\prime}}\left(t_{0}\right) A_{\pi, \pi^{\prime}, k-1}\left(t_{0}, x\right)\right) \\
+D_{\pi, \pi^{\prime}}\left(t_{0}\right)+O\left(\frac{(\log x+1)^{n}}{\log x} \exp (-C \sqrt{\log x})\right),
\end{aligned}
$$

as $x \rightarrow \infty$, for some constant $C>0, n \in \mathbb{N}$ and $t_{0} \in \mathbb{R} \backslash\{0\}$.

Remark 3.6. Representations $\pi$ and $\pi^{\prime}$ in Theorem 2.1 and Theorem 3.4 are assumed to be arbitrary, but fixed. As $t_{0} \rightarrow 0$, the representation $|\operatorname{det}|^{i t_{0}}$ formally tends to a trivial representation, hence, the condition $\pi^{\prime} \cong$ $\pi \otimes|\operatorname{det}|^{i t_{0}}$ becomes $\pi^{\prime} \cong \pi$. The statement of Theorem 2.1 agrees with this formal observation as the statement in the case when $t_{0} \rightarrow 0$ and $\pi^{\prime} \cong$ $\pi \otimes|\operatorname{det}|{ }^{i t_{0}}$ reduces to the statement when $t_{0}=0$ (i.e. $\pi^{\prime} \cong \pi$ ).

Therefore, a question of behavior of constant $\gamma_{\pi, \pi^{\prime}}(k)$ as $t_{0} \rightarrow 0$ (in a formal sense) arises naturally in this context.

When we formally let $t_{0} \rightarrow 0$ in formula (3.3), when $\pi^{\prime} \cong \pi \otimes|\operatorname{det}|^{i t_{0}}$ we may observe that

$$
\lim _{t_{0} \rightarrow 0} A_{\pi, \pi^{\prime}, k}\left(t_{0}, x\right)=\infty,
$$

hence (formally speaking) the constant $\gamma_{\pi, \pi^{\prime}}(k)$ in the case when $\pi^{\prime} \cong \pi \otimes$ $|\operatorname{det}|{ }^{i t_{0}}$ does not tend to the constant $\gamma_{\pi, \pi^{\prime}}(k)$ in the case when $\pi^{\prime} \cong \pi$.

This might come as a surprise, specially, since the Prime Number Theorem behaves continuously with respect to the formal limit $t_{0} \rightarrow 0$ and yields that

$$
\lim _{t_{0} \rightarrow 0}\left(A_{\pi, \pi^{\prime}, j}\left(t_{0}, x\right)-j ! \frac{(-1)^{j}}{\left(i t_{0}\right)^{j+1}}\right)=\frac{\log ^{j+1} x}{j+1},
$$

for all positive integers $j$.

On the other hand, in the case when $\pi^{\prime} \cong \pi \otimes|\operatorname{det}|{ }^{i t_{0}}$, for some $t_{0} \in$ $\mathbb{R} \backslash\{0\}$ we may normalize the Rankin-Selberg $L$-function $L\left(s, \pi \times \widetilde{\pi}^{\prime}\right)$ so that it possesses a pole at $s=1$, by a simple translation by $i t_{0}$. Namely, the function $L_{t_{0}}\left(s, \pi \times \widetilde{\pi}^{\prime}\right):=L\left(s+i t_{0}, \pi \times \widetilde{\pi}^{\prime}\right)$ in this case has a pole at $s=1$; the real parts of its zeros remain the same as real parts of zeros of the function $L\left(s, \pi \times \widetilde{\pi}^{\prime}\right)$ and the Dirichlet series representation of its logarithmic derivative for $\operatorname{Re} s>1$ is

$$
-\frac{L_{t_{0}}^{\prime}}{L_{t_{0}}}\left(s, \pi \times \widetilde{\pi}^{\prime}\right)=\sum_{n=1}^{\infty} \frac{\Lambda_{\pi, \pi^{\prime}}(n)}{n^{s+i t_{0}}} .
$$


Therefore, repeating the steps of the proof of Theorem 3.4 with $L_{t_{0}}(s)$ instead of $L(s)$, we immediately deduce that constants $\gamma_{\pi, \pi^{\prime}, t_{0}}(k)$ in the Laurent series expansion of $L_{t_{0}}\left(s, \pi \times \tilde{\pi}^{\prime}\right)$ around its pole $s=1$ (or, equivalently, constants arising in the Laurent series expansion of $L\left(s, \pi \times \tilde{\pi}^{\prime}\right)$ around its pole $s=$ $\left.1+i t_{0}\right)$ are given by

$$
\gamma_{\pi, \pi^{\prime}, t_{0}}(k)=\frac{(-1)^{k+1}}{k !} \lim _{x \rightarrow \infty}\left(\sum_{n \leq x} \frac{\Lambda_{\pi, \pi^{\prime}}(n)}{n^{1+i t_{0}}} \log ^{k} n-\frac{\log ^{k+1} x}{k+1}\right) .
$$

When we formally let $t_{0} \rightarrow 0$ in (3.14), we see that the formal limit as $t_{0} \rightarrow 0$ of the $k$ th constant term in the Laurent series expansion of $L^{\prime}(s) / L(s)$ around its pole $s=1+i t_{0}$ (in the case when $\pi^{\prime} \cong \pi \otimes|\operatorname{det}|^{i t_{0}}, t_{0} \in \mathbb{R} \backslash\{0\}$ ) is equal to the $k$ th constant term in the Laurent series expansion of $L^{\prime}(s) / L(s)$ around its pole $s=1$ (case $\pi^{\prime} \cong \pi$ ).

3.2. Weighted Selberg orthogonality. In this section, we give precise statements of results related to Selberg orthogonality that are immediate consequence of our main result and its proof.

First, Theorem 3.4 and the evaluation of the constant $\gamma_{\pi, \pi^{\prime}}(0)$ given in the proof of Theorem 3.4 immediately imply the following corollary.

Corollary 3.7. Let $E$ be Galois extension of $\mathbb{Q}$ of finite degree $l$ and let $\pi$ and $\pi^{\prime}$ be two irreducible automorphic unitary cuspidal representations of $G L_{m}\left(E_{\mathbb{A}}\right)$ and $G L_{m^{\prime}}\left(E_{\mathbb{A}}\right)$, respectively.

(i) Then

$$
\begin{aligned}
\sum_{n \leq x} \frac{\Lambda_{\pi, \pi^{\prime}}(n)}{n}= & \delta_{\pi, \pi^{\prime}}(0)(\log x-1)-\gamma_{\pi, \pi^{\prime}}(0) \\
& +\delta_{\pi, \pi^{\prime}}\left(t_{0}\right) \frac{x^{i t_{0}}}{i t_{0}\left(1+i t_{0}\right)}+\frac{\psi_{\pi, \pi^{\prime}}(x)}{x}+o(1),
\end{aligned}
$$

as $x \rightarrow \infty$.

(ii) Assume, additionally that at least one of $\pi$ or $\pi^{\prime}$ is self-contragredient. Then, for all integers $k \geq 0$

$$
\begin{aligned}
\sum_{n \leq x} \frac{\Lambda_{\pi, \pi^{\prime}}(n)}{n} \log ^{k} n= & (-1)^{k+1} k ! \gamma_{\pi, \pi^{\prime}}(k)+\delta(0) \frac{\log ^{k+1} x}{k+1} \\
& +\delta_{\pi, \pi^{\prime}}\left(t_{0}\right) A_{\pi, \pi^{\prime}, k}\left(t_{0}, x\right) \\
& +O\left(\log ^{k} x \exp (-C \sqrt{\log x})\right),
\end{aligned}
$$

as $x \rightarrow \infty$, where $t_{0} \in \mathbb{R} \backslash\{0\}$.

Proof. (i) Letting $c=1 / \log x$ and $T \rightarrow \infty$ in (3.4) and applying Lemma 3.2 (i) and Lemma 3.3 (i) immediately yields formula (3.15).

(ii) Straightforward from (3.3). 
REMARK 3.8. Statement (i) of the Corollary 3.7 is a generalization of Theorem 2.1. from [2] to the setting of finite Galois extensions of $\mathbb{Q}$.

In the case when $k=1$ formula (3.16) is generalization of [11, Proposition $2.2]$, and therefore a generalization of the main result from [11] in two ways. First, our method of proof enables us to obtain a unique formula for the sum in (3.16) for all $k \geq 0$ (while the result of [11] is given only for $k=1$ ). Second, our setting is slightly more general.

Furthermore, formula (3.15) enables us to remove the assumption that at least one of representations $\pi$ or $\pi^{\prime}$ is self-contragredient in the proof of Selberg orthogonality for irreducible automorphic unitary cuspidal representations of $G L_{m}\left(E_{\mathbb{A}}\right)$ and $G L_{m^{\prime}}\left(E_{\mathbb{A}}\right)$. Namely, the following proposition holds true.

Proposition 3.9. Let $E$ be Galois extension of $\mathbb{Q}$ of finite degree l and let $\pi$ and $\pi^{\prime}$ be two irreducible automorphic unitary cuspidal representations of $G L_{m}\left(E_{\mathbb{A}}\right)$ and $G L_{m^{\prime}}\left(E_{\mathbb{A}}\right)$, respectively. Assume additionally that $m, m^{\prime} \leq 4$ or, otherwise assume Hypothesis $H$ over $E$. Then

(i)

$$
\sum_{p \leq x} \frac{f_{p} \log p}{p^{f_{p}}} \sum_{v \mid p}\left(\sum_{j=1}^{m} \alpha_{\pi}(v, j)\right)\left(\sum_{l=1}^{m^{\prime}} \overline{\alpha_{\pi^{\prime}}(v, l)}\right)=\delta_{\pi, \pi^{\prime}}(0) \log x+O(1),
$$

as $x \rightarrow \infty$.

(ii)

$$
\sum_{p \leq x} \frac{f_{p}}{p^{f_{p}}} \sum_{v \mid p}\left(\sum_{j=1}^{m} \alpha_{\pi}(v, j)\right)\left(\sum_{l=1}^{m^{\prime}} \overline{\alpha_{\pi^{\prime}}(v, l)}\right)=\delta_{\pi, \pi^{\prime}}(0) \log \log x+O(1),
$$

as $x \rightarrow \infty$.

Proof. (i) When $\pi^{\prime} \equiv \pi$, then $\psi_{\pi, \pi}(x) \sim x$, as $x \rightarrow \infty$ by [10, Lemma 3.3]. This, together with Hölder inequality yields the bound $\psi_{\pi, \pi^{\prime}}(x) / x=$ $O(1)$, as $x \rightarrow \infty$. Therefore, (3.15) implies

$$
\sum_{n \leq x} \frac{\Lambda_{\pi, \pi^{\prime}}(n)}{n}=\delta_{\pi, \pi^{\prime}}(0) \log x+O(1)
$$

as $x \rightarrow \infty$. Now, the statement (i) follows from Lemma 2.2.

(ii) Application of Abel partial summation to (i) yields (ii).

\section{WEAKENING CONDITIONS ON $\pi$ AND $\pi^{\prime}$}

In this section we will prove that a weaker form of Theorem 3.4 holds true if the assumption that one of representations $\pi$ and $\pi^{\prime}$ is self-contragredient is replaced by the following assumption. 
Assumption PNT: The Prime Number Theorem (Theorem 2.1) holds true with the error term $g_{\pi, \pi^{\prime}}(x)$ such that

$$
g_{\pi, \pi^{\prime}}(x)=O\left(\frac{x}{\log ^{j} x}\right), \text { as } x \rightarrow \infty,
$$

for all $j \in \mathbb{N}$.

We will prove the following theorem.

TheOREM 4.1. Let $E$ be Galois extension of $\mathbb{Q}$ of finite degree $l$ and let $\pi$ and $\pi^{\prime}$ be two irreducible automorphic unitary cuspidal representations of $G L_{m}\left(E_{\mathbb{A}}\right)$ and $G L_{m^{\prime}}\left(E_{\mathbb{A}}\right)$, respectively such that Assumption PNT holds true. Then, for all integers $k \geq 0$

$$
\begin{aligned}
\gamma_{\pi, \pi^{\prime}}(k)= & \frac{(-1)^{k+1}}{k !}\left(\sum_{n<x} \frac{\Lambda_{\pi, \pi^{\prime}}(n)}{n} \log ^{k} n-\delta_{\pi, \pi^{\prime}}(0) \frac{\log ^{k+1} x}{k+1}\right. \\
& \left.-\delta_{\pi, \pi^{\prime}}\left(t_{0}\right) A_{\pi, \pi^{\prime}, k}\left(t_{0}, x\right)\right)+O\left(\frac{x}{\log ^{m} x}\right),
\end{aligned}
$$

for all positive integers $m$, as $x \rightarrow \infty$.

Proof. The assumption that one of representations $\pi$ and $\pi^{\prime}$ is selfcontragredient was used in the proof of Theorem 3.4 two times. First, we used it in the case when $k=0$ in order to deduce that the error term on the left hand side of $(3.6)$ is $O(\exp (-C \sqrt{\log x}))$, by applying Lemma 3.2 (ii) to (3.5). In the case when $\pi$ and $\pi^{\prime}$ are not self-contragredient, we take $c=1 / \log x$, let $T \rightarrow \infty$, apply Assumption PNT, together with Lemma 3.2 (i) to the left hand side of (3.5) and deduce that the error term on the left hand side of $(3.6)$ is $O\left(1 / \log ^{j} x\right)$, for any positive integer $j$, which is exactly the formula (4.1) for $k=0$.

Second, the assumption that one of representations $\pi$ and $\pi^{\prime}$ is self-contragredient was used in the case when $k \geq 1$ in order to deduce that the error term in $(3.12)$ is $O(\exp (-C \sqrt{\log x}))$, by applying Lemma 3.2 (ii) to (3.11). Taking $c=1 / \log x$, letting $T \rightarrow \infty$ and applying Lemma 3.2 (i) to (3.11) we get that formula (3.12) holds true with the left hand side replaced by $O\left(1 / \log ^{j} x\right)$, for any positive integer $j$.

Now, it is easy to see that formula (3.13) holds true with the error term on the left hand side replaced by $O\left(\log ^{k} x / \log ^{j} x\right)$, for any positive integer $j$. Therefore, taking $j=k+m$, from the new form of equation (3.13) we deduce that (4.1) holds true. The proof is complete.

\section{Acknowledgement}

We would like to thank the anonymous referee for his/her comments and suggestions that helped us to improve the clarity of presentation. 


\section{REFERENCES}

[1] M. Avdispahić and L. Smajlović, Euler constants for a Fuchsian group of the first kind, Acta Arith. 131 (2008), 125-143.

[2] M. Avdispahić and L. Smajlović, On the Selberg orthogonality for automorphic L-functions, Arch. Math. 94 (2010), 147-154.

[3] W. E. Briggs, Some constants associated with the Riemann zeta-function, Mich. Math. J. 3 (1955-56), 117-121.

[4] M. W. Coffey, New results on the Stieltjes constants: Asymptotic and exact evaluation, J. Math. Anal. Appl. 317 (2006), 603-612.

[5] M. W. Coffey, Series representations for the Stieltjes constants, Rocky Mountain J. Math. 44 (2014), 443-477.

[6] J. W. Cogdell, L functions and converse theorems for $G L_{n}$, Automorphic forms and applications, 97-177, IAS/Park City Math. Ser., 12, Amer. Math. Soc., Providence, RI, 2007.

[7] L. Euler, De progressionibus harmonicis observationes, Comment. acad sci. Petrop. 7 (1740), 150-161. (Opera Omnia, Series 1, Vol. 14, 87-100.)

[8] S. S. Gelbert, E. M. Lapid and P. Sarnak, A new method for lower bounds of Lfunctions, C. R. Acad. Sci. Paris 339 (2004), 91-94.

[9] S. S. Gelbert, and F. Shahidi, Boundedness of automorphic L-functions in vertical strips, J. Amer. Math. Soc. 14 (2001), 79-107.

[10] T. Gillespie and G. Ji, Prime Number Theorems for Rankin-Selberg L-functions over number fields, Sci. China Math. 54 (2011), 35-46.

[11] T. Gillespie and Y. Ye, The Prime Number Theorem and Hypothesis $H$ with lower order terms, J. Number Theory 141 (2014), 59-82.

[12] Y. Hashimoto, The Euler-Selberg constants for non-uniform lattices of rank one symmetric spaces, Kyushu J. Math. 57 (2003), 347-370.

[13] Y. Hashimoto, Y. Iijima, N. Kurokawa and M. Wakayama, Euler's constants for the Selberg and the Dedekind zeta functions, Bull. Belg. Math. Soc. Simon Stevin 11 (2004), 493-516.

[14] C. Hermite and T. J. Stieltjes, Correspondance d'Hermite et de Stieltjes, I \& II, edited by B. Baillaud and H. Bourget, Gauthier-Villars, Paris, 1905.

[15] H. Jacquet and J. A. Shalika, On Euler products and the classification of automorphic representations I, Amer. J. Math. 103 (1981), 499-558.

[16] H. Jacquet and J. A. Shalika, On Euler products and the classification of automorphic representations II. Amer. J. Math. 103 (1981), 777-815.

[17] H. Kim, Functoriality for the exterior square of $G L_{4}$ and the symmetric fourth of $G L_{2}$, J. Amer. Math. Soc. 16 (2003), 139-183.

[18] H. Kim and F. Shahidi, Cuspidality of symmetric powers with applications, Duke Math. J. 112 (2002), 177-197.

[19] C. Knessl and M. W. Coffey, An asymptotic form for the Stieltjes constants $\gamma_{k}(a)$ and for a sum $S_{\gamma}(n)$ appearing under the Li criterion, Math. Comp. 80, No. 276 (2011), $2197-2217$.

[20] X.-J. Li, The positivity of a sequence of numbers and the Riemann hypothesis, J. Number Theory 65 (1997), 325-333.

[21] J. Liu and Y. Ye, Perron's formula and the Prime Number Theorem for automorphic L-functions, Pure Appl. Math. Q. 3 (2007), 481-497.

[22] J. Liu and Y. Ye, Zeros of automorphic L-functions and noncyclic base change, in: Number Theory: Tradition and Modernization, Springer, New York, 2006, 119-152.

[23] K. Maslanka, Li's criterion for the Riemann hypothesis - numerical approach, Opuscula Math. 24 (2004), 103-114. 
[24] C. Moeglin and J.-L. Waldspurger, Le spectre résiduel de $G L(n)$, Ann. Sci. École Norm. Sup. 22 (1989), 605-674.

[25] C. J. Moreno, Explicit formulas in the theory of automorphic forms, in: Lecture Notes Math. Vol. 626, Springer, Berlin, 1977, 73-216.

[26] M. R. Murty, Problems in Analytic Number Theory, Readings in Mathematics, GTM Springer-Verlag, 2001.

[27] A. Odžak and L. Smajlović, On Li's coefficients for the Rankin-Selberg L-functions, Ramanujan J. 21 (2010), 303-334.

[28] Z. Rudnick and P. Sarnak, Zeros of principal L-functions and random matrix theory, Duke Math. J. 81 (1996), 269-322.

[29] P. Sarnak, Non-vanishing of L-functions on $\operatorname{Res}=1$, in: Contributions to automorphic forms, geometry, and number theory, Johns Hopkins Univ. Press, Baltimore, 2004, 719-732.

[30] A. Selberg, Old and new conjectures and results about a class of Dirichlet series, Collected papers, vol. II, Springer, 1991.

[31] F. Shahidi, On nonvanishing of L-functions, Bull. Amer. Math. Soc. 2, No. 3 (1980), 462-464.

[32] F. Shahidi, On certain L-functions, Amer. J. Math. 103 (1981), 297-355.

[33] F. Shahidi, Fourier transforms of intertwinting operators and Plancherel measures for $G L(n)$, Amer. J. Math. 106 (1984), 67-111.

[34] F. Shahidi, Local coefficients as Artin factors for real grups, Duke Math. J. 52 (1985), 973-1007.

[35] F. Shahidi, A proof of Langlands' conjecture on Plancherel measures, in: Complementary series for $p$-adic groups, Ann. Math. 132 (1990), 273-330.

[36] L. Smajlović, On Li's criterion for the Riemann hypotesis for the Selberg class, J. Number Theory 130 (2010), 828-851.

[37] E. C. Titchmarsh, The Theory of Functions, Second ed., Oxford University Press, Oxford, 1958.

A. Odžak

Department of Mathematics

University of Sarajevo

Zmaja od Bosne 35, 71000 Sarajevo

Bosnia and Herzegovina

E-mail: almasa@pmf.unsa.ba

L. Smajlović

Department of Mathematics

University of Sarajevo

Zmaja od Bosne 35, 71000 Sarajevo

Bosnia and Herzegovina

E-mail: lejlas@pmf.unsa.ba

Received: 18.3.2015.

Revised: 3.5.2015. 